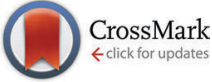

Cite this: Phys. Chem. Chem. Phys., 2016, 18, 15919

Received 19th February 2016, Accepted 22nd May 2016

DOI: $10.1039 / c 6 c p 01153 e$

www.rsc.org/pccp

\section{Revealing nanoscale optical properties and morphology in perfluoropentacene films by confocal and tip-enhanced near-field optical microscopy and spectroscopy}

\author{
Xiao Wang, ${ }^{* a}$ Katharina Broch, ${ }^{\text {bc }}$ Frank Schreiber, ${ }^{\text {b }}$ Alfred J. Meixner ${ }^{\star a}$ and \\ Dai Zhang*a
}

Combining high resolution optical microscopy and spectroscopy, we propose a novel, generally applicable and highly sensitive method for determining the local morphology in organic semiconductor thin films (e.g. perfluoropentacene (PFP)). An azimuthally or radially polarized doughnut mode (APDM or RPDM) laser beam is focused by a high numerical aperture parabolic-mirror to excite a diffraction limited volume of the PFP film with an electric field polarized either exclusively in-plane or dominantly out-of-plane (relative to the substrate). We find two distinct morphologies of thin PFP films: molecular aggregates and crystalline terraces. The well-defined dipole emission patterns observed from the molecular aggregates strongly suggest the presence of localized excitations. For both laser modes, we observe that for the PFP aggregates, the photoluminescence (PL) emission from the main electronic transition is blue-shifted by about $10 \mathrm{meV}$, as compared to that from the molecular terraces. For the $C-C$ bending modes, the $B_{3 g}$ at $1581 \mathrm{~cm}^{-1}\left(\nu_{1}\right)$ and the $A_{g}$ at $1316 \mathrm{~cm}^{-1}\left(\nu_{0}\right)$, we observe a decrease of the intensity ratio $\left(I_{\nu_{1}} / I_{\nu_{0}}\right)$ from 0.6 (terrace) to 0.15 (aggregate). Furthermore, the intensity ratios $\left(I_{\text {APDM }} / I_{\text {RPDM }}\right)$ of $\nu_{1}$ excited by different polarizations increase from 0.12 (terrace) to 0.73 (aggregate). These results indicate that the PFP molecules orient rather parallel to the substrate in the aggregates, whilst more upright in the terraces. Benefiting from the nanometer scale optical resolution offered by the tip-enhanced near-field optical method, we observe clear optical contrasts between the molecular aggregate and the terrace as well as individual layers within a terrace. Tip-enhanced optical spectra locally taken from the molecular terrace and the aggregate show similar blue-shift of the main PL peak and change in the Raman intensity with different polarizations as from the far-field assemblemeasurements, which further confirms the different molecular orientations in these two morphologies.

\section{Introduction}

Organic semiconductor thin films have attracted increasing attention for applications in flexible electronic devices. ${ }^{1}$ An important point is the chemical tunability by attaching or replacing certain functional groups. Fluorination is an important strategy, e.g., to make the compounds more stable against oxidation, to shift the relevant energy levels, or to create donor-acceptor pairs with the hydrogenated parent compound. ${ }^{2-7}$ Perfluoropentacene

\footnotetext{
${ }^{a}$ Institute of Physical and Theoretical Chemistry and LISA+, University of Tübingen, Auf der Morgenstelle 18, 72076 Tübingen, Germany. E-mail:xiao.wang@uni-tuebingen.de, alfred.meixner@uni-tuebingen.de, dai.zhang@uni-tuebingen.de

${ }^{b}$ Institute of Applied Physics, University of Tübingen, Auf der Morgenstelle 10, 72076 Tübingen, Germany

${ }^{c}$ Cavendish Laboratory, University of Cambridge, JJ Thomson Avenue,

CB3 OHE Cambridge, UK
}

(PFP, $\mathrm{C}_{22} \mathrm{~F}_{14}$ ), a derivative of the prototype system pentacene $\left(\mathrm{PEN}, \mathrm{C}_{22} \mathrm{H}_{14}\right.$ ) with exchange of the hydrogen by fluorine, is an obvious choice of study in this context. ${ }^{2,8-11}$ Compared with PEN which is a p-type semiconductor, PFP behaves as an n-type material due to the strong electronegativity of fluorine, providing the opportunity for bipolar device applications. For achieving high device performance, the fundamental properties, such as local morphology, orientation and intermolecular coupling of molecules in the films are of great interest. ${ }^{12-19}$

Although the crystal structure and the morphology of pure PFP thin films on various substrates can be investigated by X-ray diffraction (XRD) and atomic force microscopy (AFM), ${ }^{3,4,20}$ as well as other microscopic techniques, ${ }^{21}$ optical methods such as absorption, ${ }^{22-24}$ photoluminescence (PL) ${ }^{25}$ and infrared ${ }^{26}$ spectroscopy provide additional important information on the molecular energy levels and interaction in the films. Polarization-dependent micro-Raman spectroscopy provides valuable information of the 
molecular vibrations, and their modifications due to the local environment. For PEN, polarized Raman spectroscopy has been used to investigate the molecular orientation and the crystal phase of the films. ${ }^{27-29}$ Compared to linearly polarized laser beams, cylindrical vector beams, such as radially or azimuthally polarized doughnut modes (RPDM or APDM) provide distinct electric field distributions in the laser focus: a dominant out-ofplane electric field can be achieved with the RPDM whilst an exclusive in-plane electric field is produced with the APDM. Based on the polarization-dependent PL or Raman intensities, accurate and quantitative information about the molecular orientation can be obtained with diffraction-limited spatial resolution of typically half of the excitation laser wavelength. ${ }^{28,30}$ To further correlate details of the nanoscale morphology with optical properties, an optical resolution below the diffraction-limit is required which can be achieved by tip-enhanced near-field optical microscopy and spectroscopy. ${ }^{31,32}$ This technique provides images with an optical resolution down to several nanometers ${ }^{33-36}$ and sensitivities allowing for the detection of single molecules and single surface states. ${ }^{37-40}$

In this paper, we use a home-built parabolic-mirror assisted confocal and near-field optical microscope $\mathrm{e}^{41-44}$ to study the optical properties and morphology of PFP films by PL and Raman microscopy and spectroscopy with high spatial resolution. We reveal the molecular orientations in different morphologies by analysing the intensity of different Raman vibrational modes with two distinct laser excitation polarizations.

\section{Results and discussion}

The topography (Fig. 1(a) and (b)) of the PFP film shows that the film mostly forms ordered molecular terraces. The topographic line profile (Fig. 1(c)) reveals $1.5 \mathrm{~nm}$ high steps corresponding to the length of an upright-oriented single PFP molecule. ${ }^{3}$ We also observe large PFP 'aggregates', by which we mean large, anisotropic features. The aggregates are typically about several tens of nanometers high and hundreds of nanometers long, as marked by the circle in Fig. 1(b). As the orientation and stacking of molecules can be revealed from their Raman and PL spectra, we study the nature of these two distinct morphologies by polarization-dependent confocal and near-field optical microscopy and spectroscopy.

We first perform topography-correlated confocal PL and Raman imaging and spectroscopy from molecular terraces and aggregates in the PFP film. Fig. 2(a) and (b) show confocal PL images $(10 \mu \mathrm{m} \times 10 \mu \mathrm{m})$ of a PFP film excited with RPDM and APDM, respectively. In the focal volume of these polarization modes the PFP molecules are excited differently: mainly perpendicular to the substrate with RPDM and purely parallel to the substrate with APDM. In Fig. 2(a) and (b) we observe localized and diffraction-limited excitation patterns which have intensities which exceed the average PL by one order of magnitude. In general the PL excitation rate is proportional to $|\vec{E} \cdot \vec{D}|$, where $\vec{E}$ is the excitation electric field and $\vec{D}$ is the transition dipole moment. For the image obtained with RPDM we observe
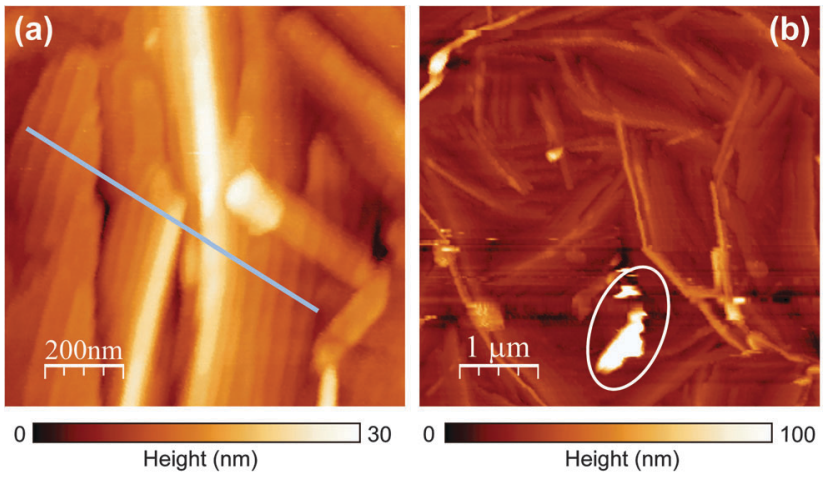

(c)

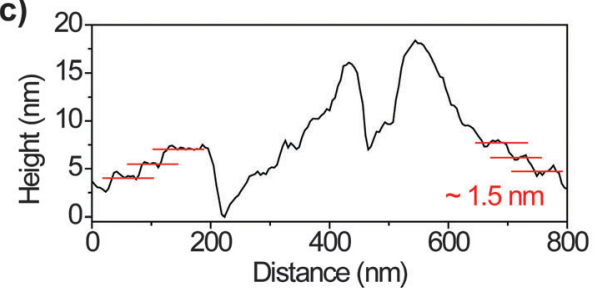

Fig. 1 ( $a$ and b) Topographic images recorded by raster scanning a sharp gold tip of the near-field optical microscope over the surface of a PFP film. (c) A respective topographic line profile that is taken from the region indicated by the line in (a). Single PFP molecular steps of about $1.5 \mathrm{~nm}$ are clearly visible.

a bright and almost circular Airy disc pattern from the structure marked by the white arrow in Fig. 2(a). This pattern reflects the projection of the transition dipole moment parallel to the surface normal of the sample. ${ }^{45}$ When RPDM is switched to APDM, the pattern changes to two parallel lobes as marked by the white arrow in Fig. 2(b). In this case, the pattern reflects the direction of the transition dipole moment projected into the sample plane which orients along the two lobes as indicated by the white arrows (on both ends). Hence for a quasi-point-light source which could originate in the present case from structural defects or local excitations, we observe distinct coffee-bean shaped patterns which allow us to determine the orientation of a transition dipole moment as in the case of an isolated molecule. ${ }^{46,47}$

Comparing the optical and the topography image (Fig. 2(c)), which are taken from the white dashed squares marked in Fig. 2(a) and (b), we clearly see that the localized excitations only occur at the aggregates and not at the terraces. In the topography images (Fig. 2(c)), both PFP aggregates and molecular terraces are observed. Fig. 2(d) shows the normalized optical intensities (upper panel, blue dots for RPDM, red dots for APDM) as well as the calculated electric field intensity distributions $\left(E^{2}\right)$ (upper panel, solid lines) and the corresponding topography (lower panel) line profiles of a PFP aggregate, which is about $80 \mathrm{~nm}$ high, $160 \mathrm{~nm}$ wide and $800 \mathrm{~nm}$ long. Due to the far-field confocal optical resolution and the weak optical contrast, the PFP molecular terraces are not clearly resolved in Fig. 2(a) and (b).

To further understand the polarization-dependent PL and Raman spectra and their relations to the local morphologies of the PFP film, spectra were recorded from different terraces and aggregates (over the $10 \mu \mathrm{m} \times 10 \mu \mathrm{m}$ area in Fig. 2) with the two polarizations. Fig. 3(a) illustrates a stack of all spectra recorded 

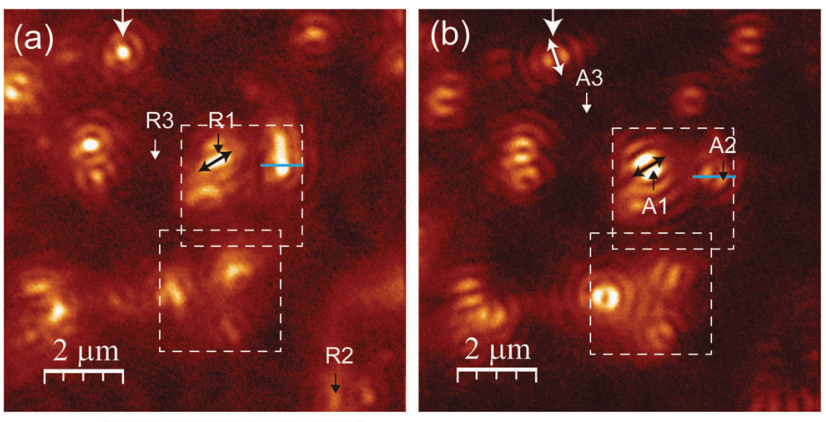

(c)



(d)

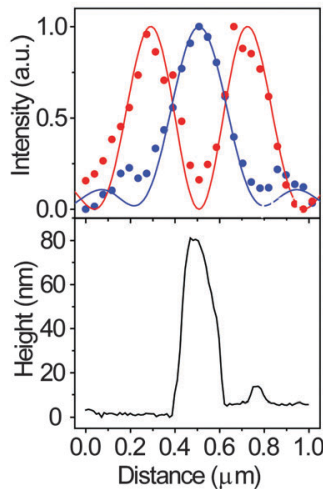

Fig. 2 ( $a$ and b) Confocal PL images of the same sections of a PFP film recorded with RPDM and APDM, respectively. Image size: $10 \mu \mathrm{m} \times 10 \mu \mathrm{m}$. (c) Topography images of the areas marked by the white dashed squares in (a) and (b). (d) The lower panel shows the topographic line profiles across the aggregate marked by a blue line in (c). In the upper panel, the dots indicate the intensity line profiles across the positions marked by the blue lines in (a) and (b); while the lines represent the calculated electric field intensity distributions $\left(E^{2}\right)$ through the focal fields in a parabolic-mirror. For the calculation, a laser wavelength of $632.8 \mathrm{~nm}$ and a Si substrate are considered. In (d), the red colour indicates the results from APDM excitation; whilst the blue one represents those from RPDM excitation.

with RPDM from the terraces to aggregates, showing increasing intensities from the bottom to the top of the picture. Three representative spectra for the high, moderate and low intensities are shown in Fig. 3(b). All the spectra consist of sharp Raman peaks and broad PL backgrounds. Clearly, the PL background dominates the optical intensity that we observed in Fig. 2(a) and (b). As shown in Fig. 3(b), The PL background of these spectra can be fitted by three Gaussian curves: one main electronic transition $\left(S_{1}\right.$ to $\left.S_{0}\right)$ in the range from $1.748 \mathrm{eV}$ to $1.736 \mathrm{eV}$ and two weak spectral shoulders at $1.865 \mathrm{eV}$ and $1.635 \mathrm{eV}$. The spectral shoulder at $1.635 \mathrm{eV}$ has been assigned to a vibronic band of the $S_{1}$ to $S_{0}$ transition. ${ }^{25}$ The dashed lines in Fig. 3(c) indicate the peak positions and show a blue-shift from the terrace to the aggregate.

Although the Raman and PL peaks from the PFP aggregates are generally stronger than from the molecular terraces as shown in Fig. 3(a), we find that the relative intensity ratios for certain Raman peaks vary significantly with the locations. To provide a clear comparison, we plot in Fig. 3(c) a series of the normalized Raman peaks of the spectra shown in Fig. 3(a) after the subtraction of the PL background. Red indicates the highest intensity; whilst blue the lowest. Three typical spectra are shown in Fig. 3(d) with an offset for clarity. The well-defined Raman peaks are in good agreement with the published results ${ }^{48}$ within

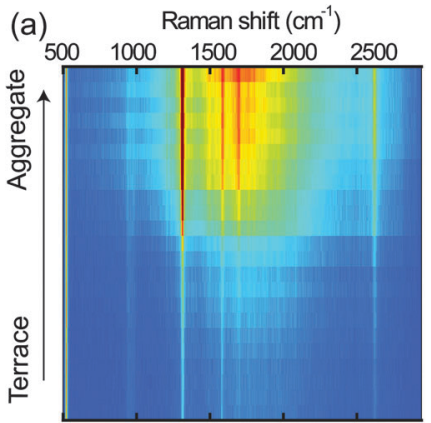

Photon energy (eV)

(b)
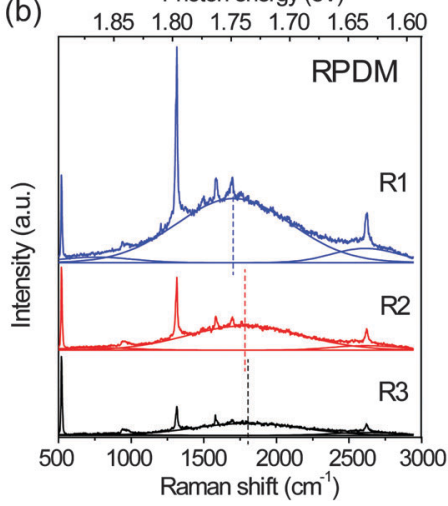

(c)

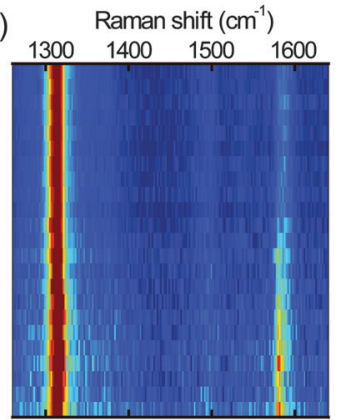

(d)

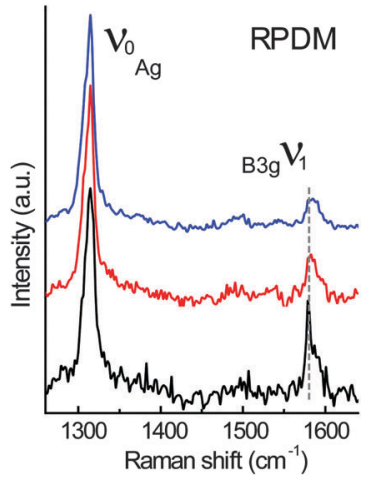

Fig. 3 (a) A series of spectra recorded with RPDM from the same area as in Fig. 2(a) and (b). (b) Three representative spectra taken from the bright (R1), moderate (R2) and dark (R3) areas by the excitation with RPDM. The Raman peaks at about $520 \mathrm{~cm}^{-1}$ and $950 \mathrm{~cm}^{-1}$ are from the Si substrate. The PL background are fitted using Gaussian function and the maxima of the fitted spectra are indicated by the dashed lines. (c) A series of Raman spectra after the subtraction of PL background in (a). The Raman spectra are normalized to the $1316 \mathrm{~cm}^{-1}$ peak. (d) Three representative Raman spectra recorded with RPDM.

the spectral resolution. The intense Raman peaks observed in the range from $1200 \mathrm{~cm}^{-1}$ to $1600 \mathrm{~cm}^{-1}$ are mainly $\mathrm{C}-\mathrm{C}$ stretching modes of the conjugated ring. ${ }^{48}$ The most prominent peak labelled as $\nu_{0}$ is located at $1316 \mathrm{~cm}^{-1}$. The peak located at $1581 \mathrm{~cm}^{-1}$ is labelled as $\nu_{1}$. For the RPDM, the Raman peak $\nu_{1}$ from the PFP terrace is more intense than that from the aggregate.

Similarly, spectra recorded with the APDM from terraces and aggregates in the $10 \mu \mathrm{m} \times 10 \mu \mathrm{m}$ area are shown as a series in the sequence from low intensity to high intensity in Fig. 4(a). Compared with the RPDM, in Fig. 4(b) the three representative spectra taken from the terraces and aggregates show similar spectral profiles and PL peak-shifts. We also show the normalized Raman spectra recorded with the APDM after the PL background subtraction in Fig. 4(c) and three representative spectra in Fig. 4(d). In contrast to the observations with the spectra recorded with RPDM, for the APDM, the intensities of the Raman peaks $\nu_{1}$ recorded from all the locations are equally weak as compared to the intense Raman peak $\nu_{0}$.

To interpret the polarization-dependent Raman intensity variations, we need to understand the Raman tensor of the vibrational modes. The chemical structure of free PFP molecule has $D_{2 \mathrm{~h}}$ symmetry. Based on the published theoretical results ${ }^{48}$ of the free PFP molecule Raman modes and the irreducible representation of $D_{2 \mathrm{~h}}$ point group, $\nu_{0}$ has an $\mathrm{A}_{\mathrm{g}}$ representation 
(a) $500 \quad 1000 \quad 1500 \quad 2000 \quad 2500$

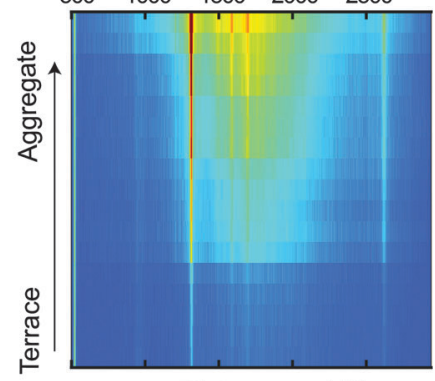

(b)


(a) Raman shift $\left(\mathrm{cm}^{-1}\right)$ (c) Raman shift $\left(\mathrm{cm}^{-1}\right)$

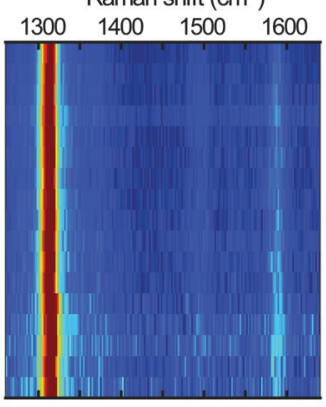

(d)



Fig. 4 (a) A series of spectra recorded with APDM. (b) Three representative spectra taken from the bright (A1), moderate (A2) and dark (A3) areas. (c) A series of normalized Raman spectra after the subtraction of PL background in (a). (d) Three representative Raman spectra recorded with APDM.

and its Raman tensor components are $x^{2}, y^{2}$ and $z^{2}$; however $\nu_{1}$ has a $\mathrm{B}_{3 \mathrm{~g}}$ representation and its Raman tensor component is $y z{ }^{49}$ Therefore, the intensity of $\nu_{1}$ is sensitive to the molecular orientations. It is known that the PFP molecule in the terrace structures (on $\mathrm{SiO}_{2} / \mathrm{Si}$ substrate) orients with the long axis almost perpendicular to the substrate surface. ${ }^{2,3}$ Since the dominant electric field in the focus of the RPDM is parallel to the substrate normal, an increase in the tilt angle of the PFP molecule with respect to the substrate normal leads to a weaker excitation, decreasing the intensity of the Raman mode. From the change of the intensity ratio of $\nu_{1}$ to $\nu_{0}$ modes, the orientation of the molecules with respect to the substrate normal can be deduced.

To obtain a comprehensive description of the molecular orientation, we analyse all spectra recorded from the sample. Fig. 5(a) shows the intensity ratios of the $\mathrm{B}_{3 \mathrm{~g}}$ Raman mode $\nu_{1}$ against the $\mathrm{A}_{\mathrm{g}}$ mode $\nu_{0}\left(I_{\nu_{1}} / I_{\nu_{0}}\right)$ at different locations as recorded with RPDM and APDM. In these plots, the different locations are represented by their total PL intensities. In general, high PL intensities correspond to PFP aggregates while low PL intensities correspond to the molecular terraces. We differentiate the experimental results from the terraces and aggregates by red dashed lines in Fig. 5(a) and (b), according to the topography and the corresponding PL intensity. Due to the exclusive in-plane excitation with APDM, the $\nu_{1}$ peaks are weak and the $\left(I_{\nu_{1}} / I_{\nu_{0}}\right)$ ratio is about 0.1 for all the locations (Fig. 5(a) right). For RPDM, the relative intensities of $\nu_{1}$ peaks vary systematically with the locations: for PFP aggregates, the $\left(I_{\nu_{1}} / I_{\nu_{0}}\right)$ ratio is about 0.15 .
For molecular terraces the PL intensity is generally lower than that from the aggregate, however, the $\left(I_{\nu_{1}} / I_{\nu_{0}}\right)$ ratio of the Raman peak increases (up to about 0.6) with the decreasing PL intensity. The changing in the $\left(I_{\nu_{1}} / I_{\nu_{0}}\right)$ ratio indicates that PFP molecule in the aggregate orients differently from it in the molecular terraces. A high $\left(I_{\nu_{1}} / I_{\nu_{0}}\right)$ ratio indicates a small molecular tilting angle with respect to the surface normal. This can be further confirmed by comparing the intensity of the $\nu_{1}$ Raman peak excited with RPDM and APDM, respectively. Fig. 5(b) shows the intensity ratio $\left(I_{\mathrm{APDM}} /\right.$ $\left.I_{\text {RPDM }}\right)$ of the Raman mode $\nu_{1}$ recorded from the terraces and aggregates. The small ratio indicates a small tilting angle which refers to the terraces; while the large ratio indicates a large tilting angle which refers to the aggregates. To demonstrate the above conclusion quantitatively, the following calculations are performed.

The Raman intensity of a certain mode is expressed by the Raman polarizability tensor $\left(\alpha_{x y z}\right)$, the electric field vector of the excitation $\left(E_{\mathrm{e}}\right)$ and scattered radiation $\left(E_{\mathrm{s}}\right)$ as $^{50}$

$$
I \propto\left|E_{\mathrm{s}}^{\mathrm{T}} \alpha_{x y z} E_{\mathrm{e}}\right|^{2}
$$

With the expression of the Raman tensor as

$$
\left[\begin{array}{lll}
0 & 0 & 0 \\
0 & 0 & f \\
0 & f & 0
\end{array}\right],
$$

the excitation intensity $\left(I_{x}, I_{y}, I_{z}\right)$ for the radial and azimuthal modes and the detection parameters $(A, B)^{28,49}$ over the scattering cone, the Raman intensity of $\mathrm{B}_{3 \mathrm{~g}}$ mode of PFP molecule which has a tilt angle $\theta$ and twist angle $\phi$ with respect to the substrate normal (Fig. 5(c)) can be calculated as in eqn (1): ${ }^{28}$

$$
I_{\mathrm{B}_{3 g}}=f^{2}\left\{\begin{array}{l}
{\left[\begin{array}{c}
A \sin ^{2} \theta\left(4 \cos ^{2} \theta \sin ^{2} \phi+\cos ^{2} \phi\right) \\
+B \sin ^{2} \phi\left(\cos ^{2} \theta-\sin ^{2} \theta\right)^{2}
\end{array}\right] I_{x}} \\
+\left[\begin{array}{c}
A \sin ^{2} \theta \cos ^{2} \phi \\
+B \cos ^{2} \theta \cos ^{2} \phi
\end{array}\right] I_{y} \\
+\left[\begin{array}{c}
A\left(\sin ^{2} \phi\left(\cos ^{2} \theta-\sin ^{2} \theta\right)^{2}+\cos ^{2} \theta \cos ^{2} \phi\right) \\
+4 B \sin ^{2} \theta \cos ^{2} \theta \sin ^{2} \phi
\end{array}\right] I_{z}
\end{array}\right\}
$$

In the calculation for the Raman intensity ratio (azimuthal against radial), the parameters are chosen as follows: $A=12.54$, $B=1.89$ for our experimental setup with an open angle of 86 degree $;^{51,52} I_{x}=I_{y}=6 \%, I_{z}=88 \%$ for the excitation intensity with RPDM; $I_{x}=I_{y}=50 \%, I_{z}=0$ for the excitation intensity with APDM. The calculated results are shown in Fig. 5(d). At small tilt angles (molecules standing upright) the ratio is much smaller, which is consistent with our experimental observations (about 0.12) at the PFP terrace. For PFP aggregates, the ratio is experimentally observed to be about 0.73 , which agrees with the calculated intensity ratio $I_{\nu_{1}} / I_{\nu_{0}}$ at larger tilting angles and a twisting angle in the range of 40 to 50 degree. 

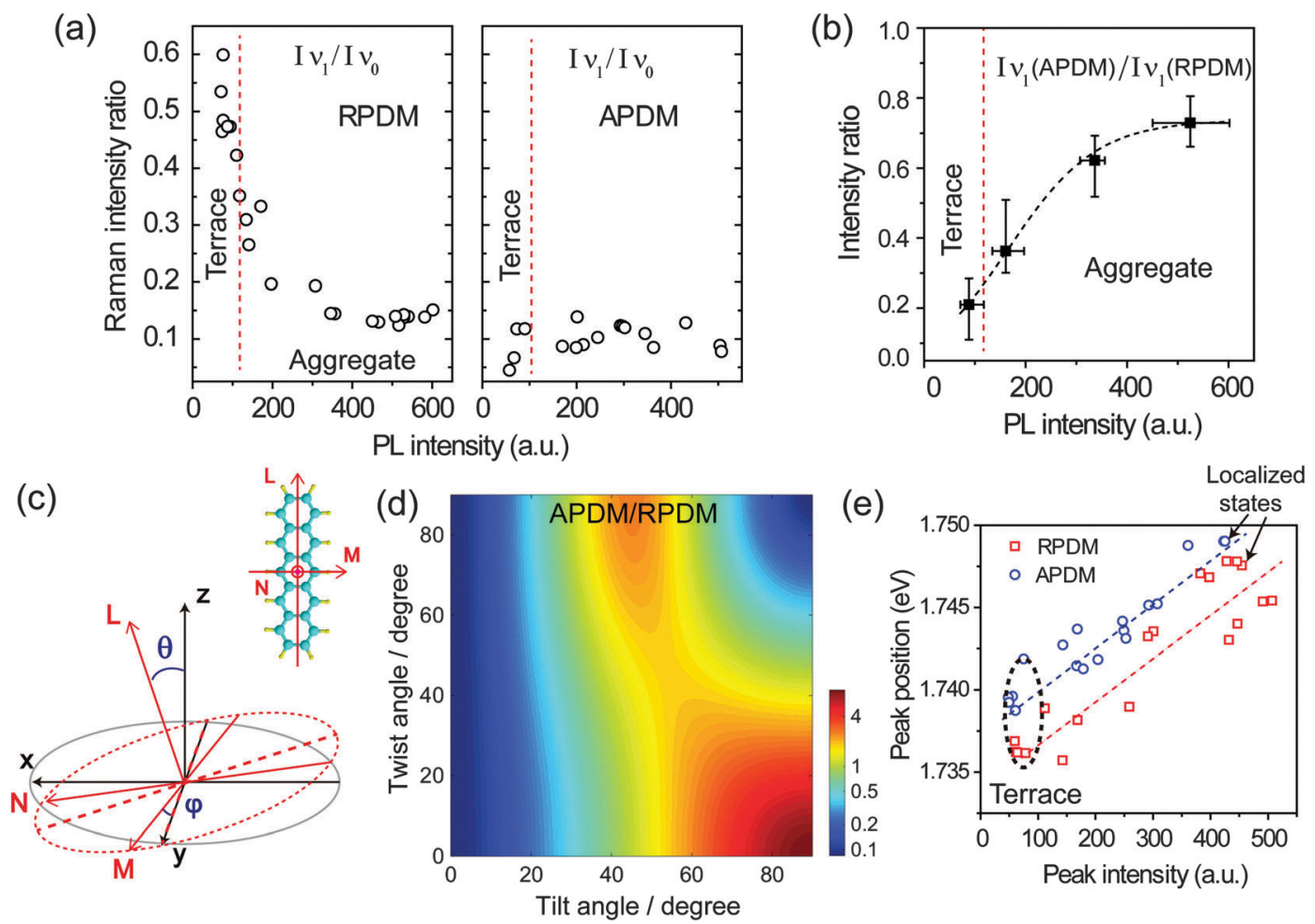

Fig. 5 (a) Intensity ratios of Raman peaks $\nu_{1}$ vs. $\nu_{0}$ at different locations excited by an APDM and RPDM, respectively. The red dashed lines differentiate the experimental results taken from the terraces and aggregates. (b) Intensity ratios of the Raman $\mathrm{B}_{3 \mathrm{~g}}$ mode $\nu_{1}\left(I_{\mathrm{APDM}} / I_{\mathrm{RPDM}}\right)$ recorded from the terraces and aggregates. The dashed line is obtained by fitting the data in (a). (c) Illustration of the tilt angle $\theta$ and the twist angle $\phi$. PFP molecule is designed with the coordinate system $(I m n)$. $l$ and $m$ denote the long and short molecular axis, respectively. $n$ denotes the direction perpendicular to the $l m$ plane. (d) Calculated intensity ratio $I_{\text {APDM }} / I_{\text {RPDM }}$ of the $B_{3 g}$ mode $\nu_{1}$ (in a logarithmic scale) as the function of the tilt angle and the twist angle. (e) Plots of PL peak positions against their intensities for the excitation with APDM (blue circle) and RPDM (red square), respectively. The dashed lines are guides to the eye.

Besides the Raman intensity ratio changes, we observe also shifts of the main PL peak for different morphologies. To provide a complete description of the behaviour, Fig. 5(e) shows the main PL emission peak, which is obtained from the spectral fitting, against the intensity at different locations in the PFP film excited with both laser modes, respectively. The peak positions and spectral widths of the other two Gaussian curves for fitting the spectral shoulders were fixed during the fitting for all different locations. For the locations where the PL intensity increases from about $60 \mathrm{k}$ counts to $500 \mathrm{k}$ counts, we observe that the PL peak position shifts by about $10 \mathrm{meV}$ to higher photon energy for both laser modes. Therefore, in general the PL emission from the PFP aggregates has higher photon energy than that from the PFP terrace. This blue-shift cannot be attributed to the film thickness-dependent HOMOLUMO transition energies of the PFP film, where a red-shift of the absorption spectrum with increasing thickness has been reported. ${ }^{23}$ Other possibilities may be considered, for example that the change in the molecular orientation at the aggregates and the terraces induces different dipole moments coupling schemes, either a $\mathrm{H}^{-}$or $\mathrm{J}$-aggregate like $\operatorname{transition}^{53}$ or $\mathrm{PL}$ emission from strained unit cells induced by the different local polymorph.

Confocal spectroscopy only allowed us to investigate how PLand Raman-spectra vary at different locations with diffraction- limited optical resolution. To optically resolve molecular terraces and the details of the aggregates, we employ near-field optical microscopy and spectroscopy to push the optical resolution down to the nanometer regime. Hence, for all near-field optical measurements a sharp gold-tip is centered in the focal volume of a RPDM for an efficient plasmon excitation. Fig. 6(a) and (b) show the simultaneously recorded topography and near-field optical images of an area with a PFP aggregate. In the topographic image (Fig. 6(a)), we observe that the PFP aggregate has a width of about $100 \mathrm{~nm}$ and a height of about $120 \mathrm{~nm}$. The corresponding near-field optical image (Fig. 6(b)) shows intense optical signals. According to the discussion based on the confocal Raman and PL spectra, the PFP molecules orient more parallel to the substrate plane in aggregates than those in the PFP terraces. As the main transition dipole moment lies along the short molecular axis, ${ }^{22}$ for PFP aggregates one would find a substantial component in the direction perpendicular to the substrate plane, which makes an efficient coupling to the tip plasmon and hence a bright near-field excited PL-signal possible.

Fig. 6(d) and (e) show the topography and the simultaneously recorded near-field optical images of an area where PFP terraces with single molecular steps are clearly resolved. The near-field optical images show optical contrast from different layers of the molecules. To demonstrate the correlation quantitatively, Fig. 6(f) shows the topographic height and optical intensity profile along one 

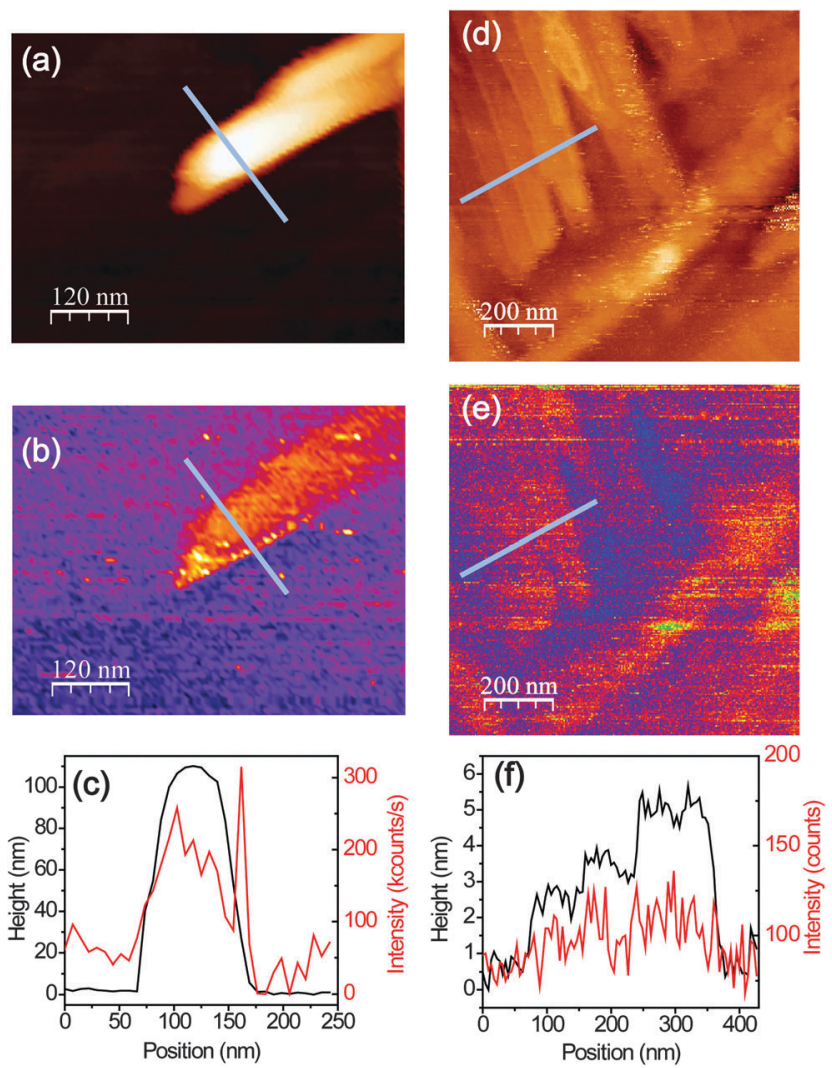

Fig. 6 (a and b) Topographic and the corresponding optical images of a PFP aggregate. (c) Topographic (black curve) and optical (red curve) line profiles along the blues lines indicated in (a) and (b). ( $d$ and e) Topographic and the corresponding optical images of the PFP terraces. (f) Topographic (black curve) and optical (red curve) line profiles along the blues lines indicated in (d) and (e). Acquisition time for each data point is $10 \mathrm{~ms}$.

line, the position of which is indicated in Fig. 6(d) and (e). Along this line the corresponding optical intensity increases from about 60 counts to 115 counts on average (130 counts at the maximum), while the height of the PFP terrace increases by only $5 \mathrm{~nm}$.

The near-field optical spectra from the PFP aggregate and the molecular terraces are shown in Fig. 7. The near-field spectrum consists of sharp Raman peaks, a PL background from the molecules and also a contribution from the gold tip. In Fig. 7 the spectra from the gold tips alone are shown as grey lines. It should be noted that two different tips are used for the measurements. The PL spectra of the gold tips are fitted by single Lorentzian curves as indicated by the red lines in Fig. 7 . Similar to the analysis for the confocal spectra, the PL background of the PFP molecules is fitted by three Gaussian curves (blue lines). After the subtraction of PL background, Raman peaks from the PFP aggregate and PFP molecular terrace are shown in Fig. 7(c) and (d), respectively. Again, we analyse the intensity ratio of Raman modes $I_{\nu_{1}} / I_{\nu_{0}}$ and the main PL peak position. From the PFP molecular terrace to the PFP aggregate, the ratio of the Raman modes $\nu_{1}$ against $\nu_{0}$ decreases from 0.69 to 0.15 , while the spectral maximum of the main PL peak shifts from $1.74 \mathrm{eV}$ to $1.75 \mathrm{eV}$. These near-field experimental results agree
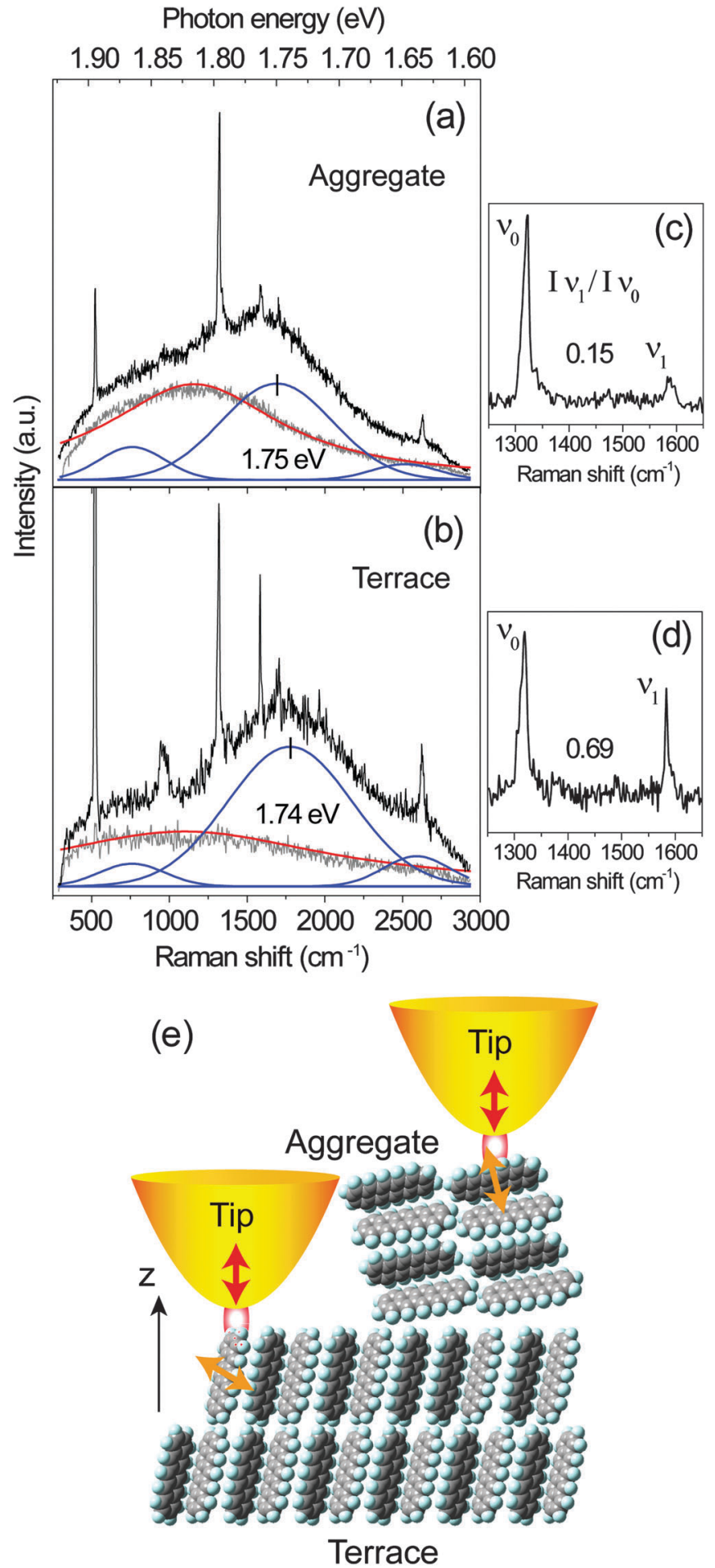

Fig. 7 ( $a$ and b) Near-field (PL and Raman) spectra recorded from the large aggregate and the molecular terrace showing in Fig. 6(a) and (b), respectively. The grey and red lines indicate the raw and fitted PL spectra of the gold tips alone. The PL of the PFP is fitted by a superposition of three blue bands at about $1.64 \mathrm{eV}, 1.74 \mathrm{eV}$ and $1.86 \mathrm{eV}$, respectively. (c and d) Raman spectra after the subtraction of the $\mathrm{PL}$ background from the spectra shown in (a) and (b), respectively. (e) Sketch of the orientation of the PFP molecules forming terraces and aggregates. The transition dipole moment (yellow arrows) orients along the short molecular axis which interacts with the optical near-field under the tip-apex (red arrows) for the excitation with RPDM. 
well with the tendency demonstrated in Fig. 5(a) however with a much higher topographical and optical resolutions. In Fig. 7(e), the interactions between a gold tip and the different locations in a PFP film, at the terrace and at the aggregate, are shown. For PFP molecules in the terraces, the main transition dipole moment is relatively parallel to the substrate plane leading to a low PL signal. ${ }^{22}$ In the molecular aggregate, the transition dipole moment orients more perpendicular to the substrate plane. The localized plasmon oscillation at the tip apex as excited by a RPDM is directed along the tip axis leading to an optical near-field under the tip apex which is mainly oriented perpendicular to the substrate plane. Hence it couples less efficiently with the molecular transition dipole moments in the molecular terraces as compared to the aggregates. Therefore, even though the PL intensity contrast is clearly visible from several molecular layers in the terraces (Fig. 6(d)-(f)), a large contrast can be observed in the near-field excited PL signal between the aggregates and the terraces (Fig. 6(a)-(c)).

\section{Experimental}

For the excitation a He-Ne laser at $\lambda=632.8 \mathrm{~nm}$ is used. The PL and Raman signals from the focal volume are collected by a parabolic mirror (numerical aperture of 0.998 in air) and are directed though two narrow band filters (in total 12 optical density) for blocking the laser line. The size (full width at the half maximum) of the diffraction limited spot (radial mode) on the sample is about $280 \mathrm{~nm}$ for the excitation with the $632.8 \mathrm{~nm}$ laser. The optical signals can be detected either by an avalanche photodiode for pixel by pixel photon counting as the sample is scanned through the laser focus; or by a spectrograph equipped with a liquid $\mathrm{N}_{2}$ cooled CCD camera for recording spectra at a fixed sample position. The spectral resolution is about $2 \mathrm{~cm}^{-1}$. For the near-field optical measurement a sharp gold tip electrochemically etched from an Au wire (diameter of $100 \mu \mathrm{m}$ ) is carefully positioned in the diffraction-limited excitation focus. A shear-force feed-back is used to station the gold tip above the sample surface at a distance of about $3 \mathrm{~nm}$. This tip is used as an optical antenna to create a highly confined, and enhanced optical near-field for the excitation; and at the same time to enhance the Stokes-shifted emission/scattering from the tipsample gap to the far-field. Topographic and near-field optical images can be obtained simultaneously by raster scanning the sample with the tip staying stationary inside the laser focus. The PFP thin films with an average thickness of $20 \mathrm{~nm}$ were grown on silicon wafers covered with a native oxide layer (thickness $\approx 2 \mathrm{~nm}$ ) at a constant substrate temperature of $30{ }^{\circ} \mathrm{C}$ under ultrahigh vacuum conditions by organic molecule beam deposition (OMBD) similar to previous studies ${ }^{54-56}$ and characterized thoroughly by complementary methods (XRD, AFM).

\section{Conclusions}

In conclusion, two distinct morphologies, ordered PFP molecular terraces and PFP aggregates are observed in PFP thin films. Based on the confocal polarization-dependent spectroscopy with RPDM, we observe a decreasing (from 0.6 to 0.15 ) intensity ratio of the $\mathrm{B}_{3 \mathrm{~g}}$ mode (at $1581 \mathrm{~cm}^{-1}$ ) against the $\mathrm{Ag}$ mode (at $1316 \mathrm{~cm}^{-1}$ ) recorded from the molecular terraces to the aggregates. It indicates that the PFP molecules orient more parallel to the substrate in the aggregates, whilst more upright in the molecular terrace. This conclusion is further confirmed by the near-field measurements with clear identification of aggregate and terrace areas and their corresponding Raman peak analysis. Furthermore, localized excitations, which could arise from either structural defects or localized excitons, appear at the aggregates and form welldefined bright dipole patterns when the sample is raster scanned through the focal field of an APDM or RPDM. We find that the PL peak position of the main electronic transition in the terraces is blue-shifted by about $10 \mathrm{meV}$ with respect to the aggregates. With the tip-enhanced near-field optical approach, we are able to push the optical resolution to sub-diffraction limit. Clear optical contrast from a single or a few PFP molecule layers is observed. Near-field spectra further confirm the blueshift of the PL peak and the decrease of the intensity ratio of the Raman modes from the PFP molecular terraces to the large aggregates. Combined with cylindrical vector beams, our confocal and near-field PL and Raman microscopy and spectroscopy provide sensitive methods to study the morphology, the orientation and the intermolecular interaction in thin molecular films.

\section{Acknowledgements}

The authors thank Reinhard Scholz for the scientific discussion and Kai Braun for improving the shear-force scanning tip runner. Financial support by the Projektförderung für Nachwuchswissenschaftler/Innen University of Tübingen and the DFG (grant ME1600/5-3, BR4869/1-1 and SCHR700/20-1) is gratefully acknowledged.

\section{References}

1 W. Brütting and C. Adachi, Physics of organic semiconductors, VCH-Wiley, Weinheim, Germany, 2012.

2 Y. Sakamoto, T. Suzuki, M. Kobayashi, Y. Gao, Y. Fukai, Y. Inoue, F. Sato and S. Tokito, J. Am. Chem. Soc., 2004, 126, 8138.

3 I. Salzmann, S. Duhm, G. Heimel, J. P. Rabe, N. Koch, M. Oehzelt, Y. Sakamoto and T. Suzuki, Langmuir, 2008, 24, 7294.

4 T. Breuer and G. Witte, Phys. Rev. B: Condens. Matter Mater. Phys., 2011, 83, 155428.

5 A. Hinderhofer and F. Schreiber, ChemPhysChem, 2012, 13, 628.

6 S. Duhm, S. Hosoumi, I. Salzmann, A. Gerlach, M. Oehzelt, B. Wedl, T. L. Lee, F. Schreiber, N. Koch, N. Ueno and S. Kera, Phys. Rev. B: Condens. Matter Mater. Phys., 2010, 81, 045418.

7 B. Milián-Medina, S. Varghese, R. Ragni, H. Boerner, E. Ortí, G. M. Farinola and J. Gierschner, J. Chem. Phys., 2011, 135, 124509.

8 Y. Inoue, Y. Sakamoto, T. Suzuki, M. Kobayashi, Y. Gao and S. Tokito, Jpn. J. Appl. Phys., 2005, 44, 3663. 
9 Y. Sakamoto, T. Suzuki, M. Kobayashi, Y. Gao, Y. Inoue and S. Tokito, Mol. Cryst. Liq. Cryst., 2006, 444, 225.

10 S.-T. Pham, Y. Kawasugi and H. Tada, Appl. Phys. Lett., 2013, 103, 143301.

11 K. Kolata, T. Breuer, G. Witte and S. Chatterjee, ACS Nano, 2014, 8, 7377.

12 I. Salzmann, S. Duhm, R. Opitz, R. L. Johnson, J. P. Rabe and N. Koch, J. Appl. Phys., 2008, 104, 114518.

13 S. D. Ha, J. Meyer and A. Kahn, Phys. Rev. B: Condens. Matter Mater. Phys., 2010, 82, 155434.

14 C. Frank, J. Novak, A. Gerlach, G. Ligorio, K. Broch, A. Hinderhofer, A. Aufderheide, R. Banerjee, R. Nervo and F. Schreiber, J. Appl. Phys., 2013, 114, 043515.

15 K. Broch, A. Gerlach, C. Lorch, J. Dieterle, J. Novak, A. Hinderhofer and F. Schreiber, J. Chem. Phys., 2013, 139, 174709.

16 K. Broch, C. Buerker, J. Dieterle, S. Krause, A. Gerlach and F. Schreiber, Phys. Status Solidi RRL, 2013, 7, 1084.

17 K. Broch, A. Aufderheide, L. Raimondo, A. Sassella, A. Gerlach and F. Schreiber, J. Phys. Chem. C, 2013, 117, 13952.

18 I. Salzmann, A. Moser, M. Oehzelt, T. Breuer, X. Feng, Z.-Y. Juang, D. Nabok, R. G. Della Valle, S. Duhm, G. Heimel, A. Brillante, E. Venuti, I. Bilotti, C. Christodoulou, J. Frisch, P. Puschnig, C. Draxl, G. Witte, K. Muellen and N. Koch, ACS Nano, 2012, 6, 10874.

19 G. B. Piland and C. J. Bardeen, J. Phys. Chem. Lett., 2015, 6, 1841.

20 S. Kowarik, A. Gerlach, A. Hinderhofer, S. Milita, F. Borgatti, F. Zontone, T. Suzuki, F. Biscarini and F. Schreiber, Phys. Status Solidi RRL, 2008, 2, 120.

21 S. Kowarik, K. Broch, A. Hinderhofer, A. Schwartzberg, J. O. Ossó, D. Kilcoyne, F. Schreiber and S. R. Leone, J. Phys. Chem. C, 2010, 114, 13061.

22 A. Hinderhofer, U. Heinemeyer, A. Gerlach, S. Kowarik, R. M. J. Jacobs, Y. Sakamoto, T. Suzuki and F. Schreiber, J. Chem. Phys., 2007, 127, 194705.

23 U. Heinemeyer, K. Broch, A. Hinderhofer, M. Kytka, R. Scholz, A. Gerlach and F. Schreiber, Phys. Rev. Lett., 2010, 104, 257401.

24 N. J. Hestand, H. Yamagata, B. Xu, D. Sun, Y. Zhong, A. R. Harutyunyan, G. Chen, H.-L. Dai, Y. Rao and F. C. Spano, J. Phys. Chem. C, 2015, 119, 22137.

25 F. Anger, J. O. Osso, U. Heinemeyer, K. Broch, R. Scholz, A. Gerlach and F. Schreiber, J. Chem. Phys., 2012, 136, 054701.

26 C. Westermeier, A. Cernescu, S. Amarie, C. Liewald, F. Keilmann and B. Nickel, Nat. Commun., 2014, 5, 4101.

27 K. Seto and Y. Furukawa, J. Raman Spectrosc., 2012, 43, 2015.

28 T. Mino, Y. Saito, H. Yoshida, S. Kawata and P. Verma, J. Raman Spectrosc., 2012, 43, 2029.

29 F. Cerdeira, M. Garriga, M. I. Alonso, J. O. Osso, F. Schreiber, H. Dosch and M. Cardona, J. Raman Spectrosc., 2013, 44, 597.
30 X. Wang, K. Broch, R. Scholz, F. Schreiber, A. J. Meixner and D. Zhang, J. Phys. Chem. Lett., 2014, 5, 1048.

31 A. Hartschuh, Angew. Chem., Int. Ed., 2008, 47, 8178.

32 C. Neacsu, S. Berweger and M. Raschke, Nanobiotechnology, 2007, 3, 172.

33 D. Zhang, U. Heinemeyer, C. Stanciu, M. Sackrow, K. Braun, L. E. Hennemann, X. Wang, R. Scholz, F. Schreiber and A. J. Meixner, Phys. Rev. Lett., 2010, 104, 056601.

34 S. Berweger, C. C. Neacsu, Y. B. Mao, H. J. Zhou, S. S. Wong and M. B. Raschke, Nat. Nanotechnol., 2009, 4, 496.

35 C. Chen, N. Hayazawa and S. Kawata, Nat. Commun., 2014, 5, 3312 .

36 A. Hartschuh, E. J. Sanchez, X. S. Xie and L. Novotny, Phys. Rev. Lett., 2003, 90, 095503.

37 J. Steidtner and B. Pettinger, Phys. Rev. Lett., 2008, 100, 236101.

38 R. Zhang, Y. Zhang, Z. C. Dong, S. Jiang, C. Zhang, L. G. Chen, L. Zhang, Y. Liao, J. Aizpurua, Y. Luo, J. L. Yang and J. G. Hou, Nature, 2013, 498, 82.

39 W. H. Zhang, B. S. Yeo, T. Schmid and R. Zenobi, J. Phys. Chem. C, 2007, 111, 1733.

40 P. C. Sevinc, X. Wang, Y. M. Wang, D. Zhang, A. J. Meixner and H. P. Lu, Nano Lett., 2011, 11, 1490.

41 P. Anger, A. Feltz, T. Berghaus and A. J. Meixner, J. Microsc., 2003, 209, 162.

42 M. Sackrow, C. Stanciu, M. A. Lieb and A. J. Meixner, ChemPhysChem, 2008, 9, 316.

43 X. Wang, D. Zhang, K. Braun, H. J. Egelhaaf, C. J. Brabec and A. J. Meixner, Adv. Funct. Mater., 2010, 20, 492.

44 X. Wang, H. Azimi, H. G. Mack, M. Morana, H. J. Egelhaaf, A. J. Meixner and D. Zhang, Small, 2011, 7, 2793.

45 J. Stadler, C. Stanciu, C. Stupperich and A. J. Meixner, Opt. Lett., 2008, 33, 681.

46 T. Zuchner, A. V. Failla and A. J. Meixner, Angew. Chem., Int. Ed., 2011, 50, 5274.

47 T. Zuchner, A. V. Failla, A. Hartschuh and A. J. Meixner, J. Microsc., 2008, 229, 337.

48 K. Fujii, C. Himcinschi, M. Toader, S. Kera, D. R. T. Zahn and N. Ueno, J. Electron. Spectrosc. Relat. Phenom., 2009, 174, 65.

49 R. Loudon, Adv. Phys., 1964, 13, 423.

50 G. Turrell, J. Raman Spectrosc., 1984, 15, 103.

51 M. A. Lieb and A. J. Meixner, Opt. Express, 2001, 8, 458.

52 C. Debus, M. A. Lieb, A. Drechsler and A. J. Meixner, J. Microsc., 2003, 210, 203.

53 K. Kolata, PhD thesis, Universität Marburg, 2014.

54 F. Schreiber, Phys. Status Solidi A, 2004, 201, 1037.

55 S. Kowarik, A. Gerlach and F. Schreiber, J. Phys.: Condens. Matter, 2008, 20, 184005.

56 K. Broch, U. Heinemeyer, A. Hinderhofer, F. Anger, R. Scholz, A. Gerlach and F. Schreiber, Phys. Rev. B: Condens. Matter Mater. Phys., 2011, 83, 245307. 